\title{
FIXED POINT THEOREMS FOR CONTRACTIVE MAPPINGS IN PROBABILISTIC MODULAR SPACES
}

\author{
SHAHNAZ JAFARI ${ }^{1, *}$, MARYAM SHAMS ${ }^{1}$ AND ASIER IBEAS ${ }^{2}$ \\ ${ }^{1}$ Department of Pure Mathematics, Shahrekord University, Iran \\ ${ }^{2}$ Department of Telecommunications and System Engineering, Faculty of Engineering, Universitat \\ Autonoma de Barcelona, 08193 Bellaterra, Cerdanyola del Valles, Barcelona, Spain \\ *Corresponding author: jafari.shahnaz@yahoo. com
}

\begin{abstract}
In this paper we introduce the concept of contractive maps and prove some related fixed point theorems in probabilistic modular spaces. In addition, we investigate the existence of common fixed points for a finite linear combination of contractive mappings. Finally, some results concerned with the convergence properties of sequences defined by contractive maps in probabilistic modular spaces are also given.
\end{abstract}

\section{INTRODUCTION}

In recent times, fixed point theory has become an important tool in pure and applied sciences, such as biology [1], chemistry [2], engineering and physics , to cite just a few. The Banach's fixed point theory, widely known as the contraction principle, is an important tool in the theory of metric spaces [3], [4]. Moreover, since the location of the fixed point can be obtained by means of an iterative process it can be implemented on a computer to find the fixed point of contraction mappings easily. The fixed point theory has been widely developed and extended to very general classes of spaces such as [5], [7], [16]. The concept of modular space was firstly introduced by Nakano [8] and it was later generalized by Musielak and Orlicz [9]. Many authors have worked ever since on the fixed point theory in modular spaces, see [10], [11], [12]. In 2009, Nourouzi introduced in [13] the notion of probabilistic modular space according to Menger's probabilistic approach [6].

Received June 20 ${ }^{\text {th }}, 2021$; accepted July 13 ${ }^{\text {th }}, 2021$; published August $12^{\text {th }}, 2021$.

2010 Mathematics Subject Classification. 47H09, 47H10.

Key words and phrases. probabilistic modular space; contrative map; fixed point; linear combination; converging sequences. (C)2021 Authors retain the copyrights of their papers, and all open access articles are distributed under the terms of the Creative Commons Attribution License. 
In this paper we introduce the concept of $\varphi$-contractive maps in probabilistic modular spaces and prove some related fixed theorems. There are no such results in probabilistic modular spaces and this paper contributes to fill in this gap. Moreover, the existence of fixed points for a finite linear combination of $\varphi$-contractive mappings in a probabilistic modular space is also investigated. Finally, we will provide some results concerned with the convergence properties of iterative sequences defined by $\varphi$-contractive maps in probabilistic modular spaces.

\section{Preliminaries}

We denote the function $\min$ by $\wedge, Z_{+}=\{z \in Z: z>0\}, Z_{0+}=Z_{+} \cup\{0\}, \mathbb{R}+=\{z \in \mathbb{R}: z>0\}$, $\mathbb{R}_{0+}=\mathbb{R}+\cup\{0\}$.We also will denote the set of all non-decreasing functions $f: \mathbb{R} \longrightarrow \mathbb{R}_{0}^{+}$satisfying $\inf _{t \in \mathbb{R}} f(t)=0$, and $\sup _{t \in \mathbb{R}} f(t)=1$ by $\Delta$. The latter properties imply that $\lim _{t \rightarrow \infty} f(t)=1$. The set of those distribution functions such that $f(0)=0$ is denoted by $\Delta^{+}$. The space $\Delta^{+}$is partially ordered by the usual pointwise ordering of functions, and has a maximal element $\epsilon_{0}$, defined by

$$
\epsilon_{0}(t)=\left\{\begin{array}{cc}
0 & t \leqslant 0 \\
1 & t>0
\end{array}\right.
$$

Definition 2.1. Let $X$ be an arbitrary vector space. A functional $\rho: X \rightarrow[0, \infty]$ is called modular if for any arbitrary $x, y \in X$ :

(ii) $\rho(x)=0$, iff $x=0$,

(ii) $\rho(\alpha x)=\rho(x)$, for every scalar $\alpha$ with $|\alpha|=1$,

(iii) $\rho(\alpha x+\beta y) \leqslant \rho(x)+\rho(y)$ for all $x, y \in X$, and $\alpha, \beta \in \mathbb{R}_{0}^{+}, \alpha+\beta=1$.

Definition 2.2. [13] A probabilistic modular space (briefly, $\mathcal{P} \mathcal{M}$-space) is a pair $(X, \mu)$ in which $X$ is a real vector space and $\mu$ is a mapping from $X$ into $\Delta$ (for $x \in X$, the function $\mu(x)$ is denoted by $\mu_{x}$, and $\mu_{x}(t)$ is the value of $\mu_{x}$ at $\left.t \in \mathbb{R}\right)$ satisfying the following conditions:

(i) $\mu_{x}(0)=0$,

(ii) $\mu_{x}(t)=1$, for all $t>0$ iff $x=0$,

(iii) $\mu_{-x}(t)=\mu_{x}(t)$, for all $x \in X$,

(iv) $\mu_{\alpha x+\beta y}(s+t) \geq \mu_{x}(s) \wedge \mu_{y}(t)$ for all $x, y \in X$, and $\alpha, \beta, s, t \in \mathbb{R}_{0}^{+}, \alpha+\beta=1$.

Definition 2.3. [13] We say that $(X, \mu)$ is $\beta$-homogeneous, where $\beta \in(0,1]$ if,

$$
\mu_{\alpha x}(t)=\mu_{x}\left(\frac{t}{|\alpha|^{\beta}}\right), \text { for every } x \in X, t>0 \text { and } \alpha \in \mathbb{R} \backslash\{0\} \text {. }
$$


Example 2.1. Let $\rho: X \rightarrow X$ be defined by $\rho(x)=\frac{1}{\alpha}|x|$, for every $\alpha \in \mathbb{R} \backslash\{0\}$. It is easy to see that $\rho$ is a modular on $X$. Define

$$
\mu_{x}(t)= \begin{cases}0 & t \leqslant 0 \\ \frac{t}{t+\rho(x)} & t>0\end{cases}
$$

for all $x \in X$. Then $(X, \mu)$ is a $\beta$-homogeneous $\mathcal{P} \mathcal{M}$-space, for $\beta=1$.

Example 2.2. Consider the real vector space $X$ with $\mu_{x}$ defined as:

$$
\mu_{x}(t)=\left\{\begin{array}{cc}
0 & t \leqslant \rho(x), \\
1 & t>\rho(x),
\end{array}\right.
$$

where $\rho$ is a modular on $X$. Then $(X, \mu)$ is a $\mathcal{P} \mathcal{M}$-space.

Definition 2.4. [13] Let $(X, \mu)$ be a $\mathcal{P} \mathcal{M}$-space.

1) A sequence $\left\{x_{n}\right\}$ in $X$ is said to be $\mu$-convergent to a point $x \in X$ and denoted by $x_{n} \longrightarrow x$, if for every $t>0$ and $r \in(0,1)$, there exists a positive integer $k$ such that $\mu_{x_{n}-x}(t)>1-r$ for all $n \geq k$. In this case, the point $x \in X$ is said to be the $\mu$-limit of the $\mu$-converging sequence $\left\{x_{n}\right\}$.

2) A sequence $\left\{x_{n}\right\}$ in $X$ is said to be $\mu$-Cauchy sequence, if for every $t>0$ and $r \in(0,1)$, there exists a positive integer $k$ such that $\mu_{x_{n}-x_{m}}(t)>1-r$ for all $n, m \geq k$.

3) The modular space $(X, \mu)$ is said to be $\mu$-complete if each $\mu$-Cauchy sequence in $X$ is $\mu$-convergent to a point of $X$.

Lemma 2.1. [13] Let $(X, \mu)$ be a $\mathcal{P} \mathcal{M}$-space. Then the $\mu$-limit of any $\mu$-convergent sequence is unique.

Lemma 2.2. [13] The operations,+ , in the $\beta$-homogeneous $\mathcal{P}$-modular space $(X, \mu)$ are continuous.

Definition 2.5. [13] Let $(X, \mu)$ and $(Y, \nu)$ be two $\mathcal{P} \mathcal{M}$-spaces. A mapping $T$ from $(X, \mu)$ to $(Y, \nu)$ is said to be sequentially $\mathcal{P} \mathcal{M}$-continuous (probabilistic modular continuous) at $x \in X$ if $T\left(x_{n}\right) \stackrel{\nu}{\longrightarrow} T(x)$ for every sequence $\left\{x_{n}\right\}$ of points in $X$ that converges to $x \in X, x_{n} \stackrel{\mu}{\longrightarrow} x$.

The definition below is introced by Sherstnev in 1963, [14].

Definition 2.6. A random normed space (briefly, $\mathcal{R} \mathcal{N}$-space) is a triple $(X, \nu, T)$ where $X$ is a vector space, $T$ is a continuous $t$-norm, and $\nu$ is a mapping from $X$ into $\Delta^{+}$(for $x \in X$, if $\nu_{x}$ denotes the value of $x \in X$, the following conditions hold:

(i) $\nu_{x}(t)=\varepsilon_{0}(t)$, for all $t>0$ iff $x=0$,

(ii) $\nu_{\alpha x}(t)=\nu_{x}\left(\frac{t}{|\alpha|}\right)$, for every $x \in X, t>0$ and $\alpha \in \mathbb{R} \backslash\{0\}$.

(iii) $\nu_{x+y}(s+t) \geq T\left(\nu_{x}(s), \nu_{y}(t)\right)$ for all $x, y \in X$ and $s, t \geq 0$.

Theorem 2.1. Let $(X, \nu, T)$ be a $\mathcal{R N}$-space with $t$-norm $T(a, b)=\min (a, b)$ for all $a, b \in X$. Then $(X, \nu)$ is a $\mathcal{P} \mathcal{M}$-space. 
Proof.

(1) $\nu_{-x}(t)=\nu_{(-1) x}(t)=\nu_{x}\left(\frac{t}{|-1|}\right)=\nu_{x}(t)$, for all $x \in X$.

(2) Let $x, y \in X, \alpha, \beta, s, t \in \mathbb{R}_{0}^{+}$and $\alpha+\beta=1$. Hence

$$
\begin{aligned}
\nu_{\alpha x+\beta y}(t) & =\nu_{\alpha x+\beta y}((\alpha+\beta) t) \\
& \geq T\left(\nu_{\alpha x}(\alpha t), \nu_{\beta y}(\beta t)\right) \\
& =T\left(\nu_{x}\left(\frac{\alpha t}{\alpha}\right), \nu_{y}\left(\frac{\beta t}{\beta}\right)\right) \\
& =T\left(\nu_{x}(t), \nu_{y}(t)\right) \\
& \geq T\left(\nu_{x}\left(\frac{t}{2}\right), \nu_{y}\left(\frac{t}{2}\right)\right) \\
& =\nu_{x}\left(\frac{t}{2}\right) \wedge \nu_{y}\left(\frac{t}{2}\right)
\end{aligned}
$$

\section{FIXED POINT THEOREMS FOR $\varphi$-CONTRACTIVE MAPPINGS}

In this section we define the notion of $\varphi$-contractive mapping in probabilistic modular spaces and prove some fixed point theorems related to this concept. Let us introduce the following definition:

Definition 3.1. A function $\varphi:[0, \infty) \rightarrow[0, \infty)$ is said to be a -function if it satisfies the following conditions:

(i) $\varphi(t)$ is continuous,

(ii) $\varphi(t)$ is strictly monotone increasing and $\varphi(t) \rightarrow \infty$ as $t \rightarrow \infty$,

(iii) $\varphi(\alpha t) \leqslant \alpha \phi(t)$, for all $\alpha \in(0,1)$ and $t \geq 0$.

It is easy to see that the condition (iii) of Definition 3.1 is equivalent to the following one: $\varphi(0)=0$.

Example 3.1. $\varphi(t)=k t^{r}$, is a simple example of $\Phi$-function for $k>0$ and $r>1$.

Lemma 3.1. A direct consequence of condition (iv) of Definition 2.2 is:

$$
\mu_{\Sigma_{i=1}^{n} \alpha_{i} x_{i}}(t) \geq \underbrace{\mu_{x_{1}}\left(\frac{t}{n}\right) \wedge \mu_{x_{2}}\left(\frac{t}{n}\right) \cdots \wedge \mu_{x_{n}}\left(\frac{t}{n}\right)}_{n},
$$

for all $x_{1}, x_{2}, \ldots, x_{n} \in X$ and $\alpha_{i}, t \in \mathbb{R}_{0}^{+}$with $\Sigma_{i=1}^{n} \alpha_{i}=1$. 
Proof. It is obtained by induction as follows:

$$
\begin{aligned}
\mu_{\sum_{i=1}^{n} \alpha_{i} x_{i}}(t) & =\mu_{\Sigma_{i=1}^{n-1} \alpha_{i} x_{i}+\alpha_{n} x_{n}}\left(\frac{(n-1) t}{n}+\frac{t}{n}\right) \\
& =\mu_{\left(\sum_{i=1}^{n-1} \alpha_{i} \frac{\Sigma_{i=1}^{n-1} \alpha_{i} x_{i}}{\sum_{i=1}^{n-1} \alpha_{i}}+\alpha_{n} x_{n}\right)}\left(\frac{(n-1) t}{n}+\frac{t}{n}\right) \\
& \geq \mu_{\frac{\sum_{i=1}^{n-1} \alpha_{i} x_{i}}{\sum_{i=1}^{n-1} \alpha_{i}}}\left(\frac{(n-1) t}{n}\right) \wedge \mu_{x_{n}}\left(\frac{t}{n}\right) \\
& \geq \underbrace{\mu_{x_{1}}\left(\frac{t}{n}\right) \wedge \mu_{x_{2}}\left(\frac{t}{n}\right) \cdots \wedge \mu_{x_{n}}\left(\frac{t}{n}\right)}_{\mathrm{n}} .
\end{aligned}
$$

Definition 3.2. Let $(X, \mu)$ be a probabilistic modular space (PM-space). A mapping $T: X \longrightarrow X$ is said to be $\varphi$-contractive if

$$
\mu_{T x-T y}(\varphi(t)) \geq \mu_{l(x-y)}\left(\varphi\left(\frac{t}{c}\right)\right)
$$

where $l, c \in(0,1)$ and $\varphi \in \Phi$.

It is easy to see that every $\varphi$-contractive map is sequentially $\mathcal{P} \mathcal{M}$-continuous. In fact, if $x_{n} \rightarrow x$, hence, for every $t>0$ and $r \in(0,1)$, there exists $N$ such that $\mu_{x_{n}-x}(t)>1-r$ for all $n \geq N$. Therefore we get $\mu_{T x_{n}-T x}(\varphi(t)) \geq \mu_{x_{n}-x}\left(\varphi\left(\frac{t}{c}\right)\right)>1-r$.

Remark 3.1. We can see that the definition 3.2 generalizes the previous ones introduced in [15].

Theorem 3.1. Let $(X, \mu)$ be a $\beta$-homogeneous $\mu$-complete $\mathcal{P} \mathcal{M}$-space and $T: X \longrightarrow X$ be a $\varphi$-contractive map. Then $T$ has a unique fixed point $x^{*} \in X$ and the iterative sequence $\left\{T^{n}\left(x_{0}\right)\right\}$, generated by the initial element $x_{0} \in X$, converges to the fixed point $x^{*} \in X$ as $n \rightarrow \infty$.

Proof. Choose $x \in X$ arbitrarily. We first prove that $\left\{T^{n}(x)\right\}$ is a $\mu$-Cauchy sequence. Let $s>0$ be given. Since $\varphi$ is continuous at 0 and $\varphi(0)=0$, we can find $t>0$ such that $\varphi(t)<s$. Hence, we have:

$$
\begin{aligned}
\mu_{T^{n} x-T^{n+p} x}(s) & \geq \mu_{T^{n} x-T^{n+p} x}(\varphi(t)) \\
& \geq \mu_{l\left(T^{n-1} x-T^{n+p-1} x\right)}\left(\varphi\left(\frac{t}{c}\right)\right) \\
& =\mu_{T^{n-1} x-T^{n+p-1} x}\left(l^{-\beta} \varphi\left(\frac{t}{c}\right)\right) \\
& \geq \mu_{T^{n-1} x-T^{n+p-1} x}\left(\varphi\left(\frac{t}{c}\right)\right) \\
& \vdots \\
& \geq \mu_{x-T^{p} x}\left(l^{-\beta} \varphi\left(\frac{t}{c^{n}}\right)\right) \\
& \geq \mu_{x-T^{p} x}\left(\varphi\left(\frac{t}{c^{n}}\right)\right) .
\end{aligned}
$$


On the other hand, we have:

$$
\begin{aligned}
\mu_{x-T^{p} x}\left(\varphi\left(\frac{t}{c^{n}}\right)\right) & =\mu_{(x-T x)+\left(T x-T^{p} x\right)}\left(\varphi\left(\frac{t}{c^{n}}\right)\right) \\
& \geq \mu_{2(x-T x)}\left(\frac{1}{2} \varphi\left(\frac{t}{c^{n}}\right)\right) \wedge \mu_{2\left(T x-T^{p} x\right)}\left(\frac{1}{2^{\prime}} \varphi\left(\frac{t}{c^{n}}\right)\right) \\
& \geq \mu_{x-T x}\left(\frac{1}{2^{\beta+1}} \varphi\left(\frac{t}{c^{n}}\right)\right) \wedge \mu_{T x-T^{p} x}\left(\frac{1}{2^{\beta+1}} \varphi\left(\frac{t}{c^{n}}\right)\right) \\
& \geq \mu_{x-T x}\left(\varphi\left(\frac{t}{2^{\beta+1} c^{n}}\right)\right) \wedge \mu_{T x-T^{p} x}\left(\varphi\left(\frac{t}{2^{\beta+1} c^{n}}\right)\right) \\
& \geq \mu_{x-T x}\left(\varphi\left(\frac{t}{2^{\beta+1} c^{n}}\right)\right) \wedge \mu_{l\left(x-T^{p-1} x\right)}\left(\varphi\left(\frac{t}{2^{\beta+1} c^{n+1}}\right)\right) \\
& =\mu_{x-T x}\left(\varphi\left(\frac{t}{2^{\beta+1} c^{n}}\right)\right) \wedge \mu_{x-T^{p-1} x}\left(l^{-\beta} \varphi\left(\frac{t}{2^{\beta+1} c^{n+1}}\right)\right) \\
& \geq \mu_{x-T x}\left(\varphi\left(\frac{t}{2^{\beta+1} c^{n}}\right)\right) \wedge \mu_{x-T^{p-1} x}\left(\varphi\left(\frac{t}{2^{\beta+1} c^{n+1}}\right)\right) .
\end{aligned}
$$

By induction we get:

$$
\begin{aligned}
\mu_{x-T^{p} x}\left(\varphi\left(\frac{t}{c^{n}}\right)\right) & \geq \mu_{x-T x}\left(\varphi\left(\frac{t}{2^{\beta+1} c^{n}}\right)\right) \wedge \mu_{x-T x}\left(\varphi\left(\frac{t}{2^{2(\beta+1)} c^{n+1}}\right)\right) \wedge \cdots \\
& \wedge \mu_{x-T x}\left(\varphi\left(\frac{t}{2^{p(\beta+1)} c^{n+p-1}}\right)\right) .
\end{aligned}
$$

According to property $(i i)$ of $\Phi$-function and since $\mu(\infty)=1$, from $(3.4)$ and (3.6) we get $\lim _{n \rightarrow \infty} \mu_{T^{n} x-T^{n+p} x}(s)=$ 1. Since $X$ is $\mu$-complete, there exists $x^{*} \in X$ with $\lim _{n \rightarrow \infty} T^{n} x^{*}=x^{*}$.

We will prove now that $x^{*}$ is a fixed point of $T$. The $\varphi$-contractivity of $T$ yields sequentialy $\mathcal{P} \mathcal{M}$-continuity. Therefore, $x^{*}=\lim _{n \rightarrow \infty} T^{n+1} x^{*}=\lim _{n \rightarrow \infty} T\left(T^{n} x^{*}\right)=T x^{*}$; i.e $x^{*}$ is a fixed point of $T$. In order to prove that the fixed point if unique, assume that there exists another fixed point $y^{*} \in X$ such that $y^{*}=T y^{*}$. Hence, $T^{n} x^{*}=x^{*}$ and $T^{n} y^{*}=y^{*}$, and there exists $t_{1}>0$ such that $\mu_{y^{*}-x^{*}}\left(t_{1}\right)=a<1$. Then,

$$
\begin{aligned}
a=\mu_{y^{*}-x^{*}}\left(t_{1}\right) & \geq \mu_{y^{*}-x^{*}}(\varphi(t)) \\
& =\mu_{T^{n} y^{*}-T^{n} x^{*}}(\varphi(t)) \\
& \geq \mu_{y^{*}-x^{*}}\left(l^{-n \beta} \varphi\left(\frac{t}{c^{n}}\right)\right) .
\end{aligned}
$$

Letting $n \rightarrow \infty$ in (3.7), according the property $(i i)$ of $\Phi$-function and since $\mu(\infty)=1$, we get $a \geq 1$, that is contradiction. Therefore $y^{*}=x^{*}$.

The following Theorem shows that a linear combination of a family of $\varphi$-contrative mappings possesing a common fixed point has a fixed point and it can be calculated by using an interative process.

Theorem 3.2. Let $(X, \mu)$ be a $\beta$-homogeneous $\mu$-complete $\mathcal{P} \mathcal{M}$-space and $f_{i}: X \longrightarrow X(i=1,2, \cdots, m)$ be a finite family of $\varphi$-contractive maps for $\varphi \in \Phi$ and $c \in\left(0, \frac{1}{m}\right)$. Define $f=\sum_{i=1}^{m} \lambda_{i} f_{i}$, where $\lambda_{i} \in[0,1]$, $\sum_{i=1}^{m} \lambda_{i}=1$. Then $f$ has fixed point $x^{*} \in X$, which is common to each linear operator's one and the iterative sequence $\left\{f^{n}(x)\right\}$ defined by the initial element $x_{0} \in X$, converges to $x^{*} \in X$. 
Proof. Since $f_{i}$ have a common fixed point $x^{*} \in X$, then:

$$
f\left(x^{*}\right)=\lambda_{1} f_{1}\left(x^{*}\right)+\lambda_{2} f_{2}\left(x^{*}\right)+\ldots+\lambda_{m} f_{m}\left(x^{*}\right)=\left(\lambda_{1}+\lambda_{2}+\ldots+\lambda_{m}\right) x^{*}=x^{*},
$$

This means that $x^{*}$ is a fixed of $f$ (and common to ech operator's fixed point). Now we prove that $f$ is a $\varphi$-contractive map. We have:

$$
\begin{aligned}
\mu_{f x-f y}(\varphi(t)) & =\mu_{\sum_{i=1}^{m} \lambda_{i} f_{i} x_{i}-\sum_{i=1}^{m} \lambda_{i} f_{i} y}(\varphi(t)) \\
& \geq \underbrace{\mu_{f_{1} x-f_{1} y}\left(\frac{1}{m} \varphi(t)\right) \wedge \mu_{f_{2} x-f_{2} y}\left(\frac{1}{m} \varphi(t)\right) \wedge \cdots \wedge \mu_{f_{n} x-f_{n} y}\left(\frac{1}{m} \varphi(t)\right)}_{\mathrm{m}} \\
& \geq \underbrace{\mu_{f_{1} x-f_{1} y}\left(\varphi\left(\frac{t}{m}\right)\right) \wedge \mu_{f_{2} x-f_{2} y}\left(\varphi\left(\frac{t}{m}\right)\right) \wedge \cdots \wedge \mu_{f_{n} x-f_{n} y}\left(\varphi\left(\frac{t}{m}\right)\right)}_{\mathrm{m}} \\
& \geq \mu_{l(x-y)}\left(\varphi\left(\frac{t}{m c}\right)\right) \wedge \mu_{l(x-y)}\left(\varphi\left(\frac{t}{m c}\right)\right) \wedge \cdots \wedge \mu_{l(x-y)}\left(\varphi\left(\frac{t}{m c}\right)\right) \\
& \geq \mu_{l(x-y)}\left(\varphi\left(\frac{t}{m c}\right)\right) \\
& =\mu_{l(x-y)}\left(\varphi\left(\frac{t}{k}\right)\right),
\end{aligned}
$$

where $k \in(0,1)$. Hence, $f$ is $\varphi$-contractive and according to Theorem 3.1 , the sequence $\left\{f^{n}\left(x_{0}\right)\right\}$ converges to the fixed point $x^{*} \in X$ for any arbitrary initial element $x_{0}$.

The subsequent results are concerned with the convergence properties of $\varphi$-contractive maps. $\mathcal{P} \mathcal{M}$-space.

Lemma 3.2. The following property hold:

If $T_{n}: X \rightarrow X, \forall n \in Z_{+}$are continuous and $\left\{T_{n}\right\}$ uniformly converges to $\{T\}$, then $\left\{T_{n}^{m}\right\}$ uniformly converge to $\left\{T^{m}\right\}, \forall m \in Z_{+}$.

Proof. We prove these properties with induction. Assume that $\left\{T_{n}^{j}\right\}$ converge to $\left\{T^{j}\right\}$, as $n \rightarrow \infty$, for all $1 \leq j \leq m$ and for any given $m \in Z_{+}$. We have:

$$
\mu_{T_{n}^{j}\left(T_{n} x\right)-T^{j}(T x)}(t) \geq \mu_{2\left(T_{n}^{j}\left(T_{n} x\right)-T^{j}\left(T_{n} x\right)\right)}\left(\frac{t}{2}\right) \wedge \mu_{2\left(T^{j}\left(T_{n} x\right)-T^{j}(T x)\right)}\left(\frac{t}{2}\right)
$$

since $T_{n}: X \rightarrow X$ is continuous and $\left\{T_{n}^{j}\right\}$ converge to $\left\{T^{j}\right\}$, there exists a big enough $n$ such that $\mu_{T^{j}\left(T_{n} x\right)-T^{j}(T x)}\left(\frac{t}{2}\right)>1-\lambda$ and $\mu_{T_{n}^{j}\left(T_{n} x\right)-T^{j}\left(T_{n} x\right)}\left(\frac{t}{2}\right)>1-\lambda$, for any given $\lambda \in(0,1)$. Thus, from (3.10), we have

$$
\mu_{T_{n}^{j+1} x-T^{j+1} x}(t)>(1-\lambda) \wedge(1-\lambda)=1-\lambda
$$

Thus, $T_{n}^{j+1}$ converge to $T^{j+1}$ as $n \rightarrow \infty$, for all $1 \leqslant j \leqslant m$.

Theorem 3.3. Let $(X, \mu)$ be a $\beta$-homogeneous $\mu$-complete $\mathcal{P} \mathcal{M}$-space and $\left\{T_{n}\right\}$ be a sequence of sequentially $\mathcal{P} \mathcal{M}$-continuous operators with $\operatorname{Fix}\left(T_{n}\right)=\left\{x_{n}^{*}\right\}$, such that: 
(i) $\left\{T_{n}\right\}$ uniformly converge to $T$ for some $T: X \longrightarrow X$.

(ii) $T$ is $\varphi$-contractive, with $T\left(x^{*}\right)=x^{*}$.

Then $\left\{x_{n}^{*}\right\} \rightarrow x^{*}$.

Proof. According to the definition of convergence in $\mathcal{P} \mathcal{M}$-space, we show that $\lim _{n \rightarrow \infty} \mu_{x_{n}^{*}-x^{*}}(t)=1$, for every $t>0$. In this way, we have:

$$
\begin{aligned}
\mu_{x_{n}^{*}-x^{*}}(\varphi(t)) & =\mu_{T_{n}^{m} x_{n}^{*}-T^{m} x^{*}}(\varphi(t)) \\
& \geq \mu_{2\left(T_{n}^{m} x_{n}^{*}-T^{m} x_{n}^{*}\right)}\left(\varphi\left(\frac{t}{2}\right)\right) \wedge \mu_{2\left(T^{m} x_{n}^{*}-T^{m} x^{*}\right)}\left(\varphi\left(\frac{t}{2}\right)\right) \\
& \geq \mu_{2\left(T_{n}^{m} x_{n}^{*}-T^{m} x_{n}^{*}\right)}\left(\varphi\left(\frac{t}{2}\right)\right) \wedge \mu_{x_{n}^{*}-x^{*}}\left(\varphi\left(\frac{t}{2^{\beta+1} c^{m}}\right)\right), \quad \forall n, m \in Z_{0^{+}},
\end{aligned}
$$

If we take the limit $m \rightarrow \infty$ in (3.11) we get $\lim _{n \rightarrow \infty} \mu_{x_{n}^{*}-x^{*}}(\varphi(t))=1$. Thus, $\left\{x_{n}^{*}\right\} \rightarrow\left\{x^{*}\right\}$.

Theorem 3.4. Let $(X, \mu)$ be a $\beta$-homogeneous $\mu$-complete $\mathcal{P} \mathcal{M}$-space and $\left\{T_{n}\right\}$ be a sequence of $\varphi$-contractive operators $T_{n}: X \rightarrow X$ for some $l, c \in(0,1), \varphi \in \Phi$ with Fix $\left(T_{n}\right)=\left\{x_{n}^{*}\right\}$. Moreover, let $T: X \longrightarrow X$ be a $\varphi$-contractive mapping with $\operatorname{Fix}(T)=\left\{x^{*}\right\}$. Assume the following properties hold:

(a) $\left\{T_{n}\right\}$ converge to $T$,

(b) There exists a subsequence $\left\{x_{n_{m}}^{*}\right\}$ of $\left\{x_{n}^{*}\right\}$, converging to a point $z \in X$.

Then $z=x^{*}$ and the iterated sequence generated by $x_{n+1}=T_{n} x_{n}$ converges to the fixed point $x^{*}$, for any given $x_{0} \in X$ and $n \in Z_{+}$

Proof. We first prove that $\left\{x_{n_{m}}^{*}\right\}$ converge to $x^{*}$. Proceed by assuming, since $\left\{x_{n_{m}}^{*}\right\} \rightarrow\{z\}$ and $\left\{T_{n}\right\} \rightarrow T$, for any given $\delta \in(0,1)$ and $t>0$ there exists $N_{1}\left(\in Z_{0+}\right)=N_{1}(\delta, t)$ such that for $n, m \geq N_{1}, \mu_{x_{n_{m}}^{*}-z}(\varphi(t))>$ $1-\delta$ and $\mu_{T_{n_{m}} z-T z}(\varphi(t))>1-\delta$, where $\varphi \in \Phi$. Therefore,

$$
\begin{aligned}
\mu_{x_{n_{m}}^{*}-T z}(\varphi(t)) & =\mu_{T_{n_{m}} x_{n_{m}}^{*}-T z}(\varphi(t)) \\
& \geq \mu_{2\left(T_{n_{m}} x_{n_{m}}^{*}-T_{n_{m}} z\right)}(\varphi(t)) \wedge \mu_{2\left(T_{n_{m}} z-T z\right)}(\varphi(t)) \\
& \geq \mu_{x_{n_{m}}^{*}-z}\left(\varphi\left(\frac{t}{2^{\beta} c}\right)\right) \wedge \mu_{T_{n_{m}} z-T z}\left(2^{-\beta} \varphi(t)\right) \\
& \geq(1-\delta) \wedge(1-\delta) \\
& =(1-\delta) .
\end{aligned}
$$


This result means that $\left\{x_{n_{m}}^{*}\right\}$ converges to $T z$. Hence, by the uniqueness of the limit, we get $T z=z$ implying that $z=x^{*}$. Also for $\alpha \in(0,2 c)$ we have:

$$
\begin{aligned}
\mu_{x_{n+1}-x^{*}}(\varphi(t)) & =\mu_{T_{n} x_{n}-T x^{*}}(\varphi(t)) \\
& \geq \mu_{2\left(T_{n} x_{n}-T_{n} x^{*}\right)}(\alpha \varphi(t)) \wedge \mu_{2\left(T_{n} x^{*}-T x^{*}\right)}((1-\alpha) \varphi(t)) \\
& \geq \mu_{x_{n}-x^{*}}\left(\varphi\left(\frac{\alpha t}{2^{\beta} c}\right)\right) \wedge \mu_{2\left(T_{n} x^{*}-T x^{*}\right)}((1-\alpha) \varphi(t)) .
\end{aligned}
$$

On the other hand:

$$
\mu_{x_{n}-x^{*}}\left(\varphi\left(\frac{\alpha t}{2^{\beta} c}\right)\right) \geq \mu_{x_{n-1}-x^{*}}\left(\varphi\left(\frac{\alpha^{2} t}{2^{2(\beta+1)} c^{2}}\right)\right) \wedge \mu_{2\left(T_{n} x^{*}-T x^{*}\right)}\left((1-\alpha) \varphi\left(\frac{\alpha t}{2^{\beta} c}\right)\right)
$$

By induction from (3.13) and (3.14), we have

$$
\begin{aligned}
\mu_{x_{n+1}-x^{*}}(\varphi(t)) & \geq \mu_{x_{0}-x^{*}}\left(\varphi\left(\frac{\alpha^{n} t}{2^{n(\beta+1)} c^{n}}\right)\right) \wedge \mu_{2\left(T_{n} x^{*}-T x^{*}\right)}((1-\alpha) \varphi(t)) \\
& \wedge \mu_{2\left(T_{n} x^{*}-T x^{*}\right)}\left((1-\alpha) \varphi\left(\frac{\alpha t}{2^{\beta} c}\right)\right) \wedge \cdots \\
& \wedge \mu_{2\left(T_{n} x^{*}-T x^{*}\right)}\left((1-\alpha) \varphi\left(\frac{\alpha^{n} t}{2^{n(\beta+1)} c^{n}}\right)\right) .
\end{aligned}
$$

Letting $n \rightarrow \infty$ in $(3.15)$ we get $\lim \mu_{x_{n+1}-x^{*}}(\varphi(t)) \geq 1$, i.e $\left\{x_{n}\right\} \rightarrow x^{*}$.

Theorem 3.5. Let $(X, \mu)$ be a $\beta$-homogeneous $\mu$-complete $\mathcal{P} \mathcal{M}$-space, and $\left\{T_{n}\right\}$ be a sequence of operators such that $\left\{T_{n}\right\}$ are $\varphi$-contractive for some $l, c \in(0,1)$ and $\varphi \in \Phi$. Assume that $\left\{T_{n}\right\}$ converge to $T$ for some $T: X \longrightarrow X$. Then the following properties hold:

(a) $T$ is $\varphi$-contractive for some $c \in\left(0, \frac{1}{4}\right)$,

(b) $\left\{x_{n}^{*}\right\} \rightarrow x^{*}$, where Fix $\left(T_{n}\right)=\left\{x_{n}^{*}\right\}, \forall n \in Z_{+}$, and Fix $(T)=\left\{x^{*}\right\}$,

(c) The iterative sequence generated by $x_{n+1}=T_{n} x_{n}$ converges to $x^{*}$, for any given $x_{0} \in X$ arbitrary and $n \in Z_{+}$.

Proof. First we prove that $T$ is $\varphi$-contractive. For any $x, y \in X$ we have:

$$
\mu_{T_{n} x-T_{n} y}(\varphi(t)) \geq \mu_{l(x-y)}\left(\varphi\left(\frac{t}{c}\right)\right)
$$

Additionally,

$$
\begin{aligned}
\mu_{T x-T y}(\varphi(t)) & =\mu_{T x-T_{n} x+T_{n} x-T y}(\varphi(t)) \\
& \geq \mu_{2\left(T x-T_{n} x\right)}((1-\alpha) \varphi(t)) \wedge \mu_{2\left(T_{n} x-T y\right)}(\alpha \varphi(t)) \\
& \geq \mu_{T x-T_{n} x}\left(\frac{\varphi((1-\alpha) t)}{2^{\beta}}\right) \wedge \mu_{T_{n} x-T y}\left(\frac{\varphi(\alpha t)}{2^{\beta}}\right) .
\end{aligned}
$$


On the other hand

$$
\begin{aligned}
\mu_{T_{n} x-T y}\left(\frac{\varphi(\alpha t)}{2^{\beta}}\right) & \geq \mu_{2\left(T_{n} x-T_{n} y\right)}\left(\frac{\varphi(\alpha t)}{2^{\beta+1}}\right) \wedge \mu_{2\left(T_{n} y-T y\right)}\left(\frac{\varphi(\alpha t)}{2^{\beta+1}}\right) \\
& \geq \mu_{T_{n} x-T_{n} y}\left(\varphi\left(\frac{\alpha t}{2^{2(\beta+1)}}\right)\right) \wedge \mu_{T_{n} y-T y}\left(\frac{\varphi(\alpha t)}{2^{2(\beta+1)}}\right) \\
& \geq \mu_{l(x-y)}\left(\varphi\left(\frac{c^{-1} \alpha t}{2^{2(\beta+1)}}\right)\right) \wedge \mu_{T_{n} y-T y}\left(\frac{\varphi(\alpha t)}{2^{2(\beta+1)}}\right) .
\end{aligned}
$$

By using equation (3.17) and (3.18) we have

$$
\mu_{T x-T y}(\varphi(t)) \geq \mu_{T x-T_{n} x}\left(\frac{\varphi((1-\alpha) t)}{2^{\beta}}\right) \wedge \mu_{l(x-y)}\left(\varphi\left(\frac{c^{-1} \alpha t}{2^{2(\beta+1)}}\right)\right) \wedge \mu_{T_{n} y-T y}\left(\frac{\varphi(\alpha t)}{2^{2(\beta+1)}}\right) .
$$

Letting $n \rightarrow \infty$ in (3.19), we get

$$
\begin{aligned}
\mu_{T x-T y}(\varphi(t)) & \geq 1 \wedge \mu_{l(x-y)}\left(\varphi\left(\frac{c^{-1} \alpha t}{2^{2(\beta+1)}}\right)\right) \wedge 1 \\
& =\mu_{l(x-y)}\left(\varphi\left(\frac{c^{-1} t}{2^{2(\beta+1)}}\right)\right) \\
& \geq \mu_{l(x-y)}\left(\varphi\left(\frac{t}{k}\right)\right),
\end{aligned}
$$

where $k \in\left(0, \frac{1}{4}\right)$. Hence $T$ is $\varphi$-contractive. Finally, according to Theorem $3.3,\left\{x_{n}^{*}\right\}$ converges to $x^{*}$ and by the same method of proof of Theorem 3.4, $\left\{x_{n}\right\}$ converges to $x^{*}$.

Remark 3.2. Every probabilistic modular space $(X, \mu)$ induces a probabilistic metric space $(X, F, \wedge)$ with $F: X \times X \rightarrow \Delta$ via $F_{x, y}=\mu_{x-y}$ for all $x, y \in X$.

\section{NumericAl EXAMPLES}

In this section we present some numerical examples in order to illustrate the main results discussed in the previous sections.

Example 4.1. Let $X=\mathbb{R}$ and $\mu_{x}(t)=\frac{t}{t+\rho(x)}, x, y \in X, t>0$, where $\rho(x)=|x|$ is a modular functional on $X$. Define a mapping $f: \mathbb{R} \rightarrow \mathbb{R}$ by $f(x)=\frac{x}{8}$ for all $x \in \mathbb{R}$. Let $\varphi(t)=2 t^{2}$. Then $f$ is $\varphi$-contractive with the constants $l=\frac{1}{2}$ and $c \geq \frac{1}{2}$. Indeed, for $x, y \in \mathbb{R}$, we have

$$
\mu_{f x-f y}(\varphi(t))=\frac{2 t^{2}}{2 t^{2}+\frac{1}{8}|x-y|}, \quad \mu_{l(x-y)}\left(\varphi\left(\frac{t}{c}\right)\right)=\frac{\frac{2 t^{2}}{c^{2}}}{\frac{2 t^{2}}{c^{2}}+\frac{1}{2}|x-y|} .
$$

It is easy to see that

$$
\frac{2 t^{2}}{2 t^{2}+\frac{1}{8}|x-y|} \geq \frac{\frac{2 t^{2}}{c^{2}}}{\frac{2 t^{2}}{c^{2}}+\frac{1}{2}|x-y|}
$$

for all $c \in\left[\frac{1}{2}, 1\right)$. Accordingly, $f$ is $\varphi$-contractive and it has a unique fixed point, as predicted by Theorem 3.1. In addition, it is easy to check that $x=0$ is the fixed point of $f$. 
Example 4.2. Let $X=\mathbb{R}$ and $\mu_{x}(t)=\frac{t}{t+\rho(x)}, x, y \in X, t>0$, where $\rho(x)=|x|$ is a modular functional on $X$. Define the mappings $f_{i}: \mathbb{R} \rightarrow \mathbb{R}$ by $f_{1}(x)=\frac{x}{4}$ and $f_{2}(x)=\frac{x}{2}$ for all $x \in \mathbb{R}$. Let $\varphi(t)=t, l=\frac{2}{3}$ and $c=\frac{3}{4}$. Define $f=\frac{1}{2} f_{1}+\frac{1}{2} f_{2}$. We can see that $f_{1}$ and $f_{2}$ are $\varphi$-contractive maps. We prove that $f(x)=\frac{1}{2} f_{1} x+\frac{1}{2} f_{2} x=\frac{3 x}{8}$ is $\varphi$-contractive.

$$
\mu_{f x-f y}(\varphi(t))=\frac{t}{t+\frac{3}{8}|x-y|}, \quad \mu_{l(x-y)}\left(\varphi\left(\frac{t}{c}\right)\right)=\frac{\frac{4}{3} t}{\frac{4}{3} t+\frac{2}{3}|x-y|},
$$

Thus,

$$
\frac{t}{t+\frac{3}{8}|x-y|} \geq \frac{\frac{4}{3} t}{\frac{4}{3} t+\frac{2}{3}|x-y|}
$$

Consequently, $f$ is a $\varphi$-contractive map and it has a unique fixed point, as predict by Theorem 3.2. It is easy to check that $x=0$ is the fixed point of $f$.

Example 4.3. Let $X=\mathbb{R}$ and $\mu_{x}(t)=\frac{t}{t+\rho(x)}, x, y \in X, t>0$, where $\rho(x)=|x|$ is a modular functional on $X$. Let $\varphi(t)=t$ and define $T_{n} x_{n}=\frac{(n+1) x_{n}}{(2 n+3)\left(1+x_{n}^{2}\right)}$. We show that $T_{n}$ is $\varphi$-contractive. We have:

$$
\mu_{T_{n} x-T_{n} y}(\varphi(t))=\frac{t}{t+\frac{(n+1)|x-y|}{(2 n+3)\left(1+x^{2}\right)(1+y)}}=\frac{(2 n+3)\left(1+x^{2}\right)(1+y) t}{(2 n+3)\left(1+x^{2}\right)(1+y) t+(n+1)|x-y|},
$$

and

$$
\mu_{l(x-y)}\left(\varphi\left(\frac{t}{c}\right)\right)=\frac{\frac{t}{c}}{\frac{t}{c}+l|x-y|}=\frac{t}{t+l c|x-y|} .
$$

So the condition (3.3) becomes:

$$
\frac{(2 n+3)\left(1+x^{2}\right)(1+y) t}{(2 n+3)\left(1+x^{2}\right)(1+y) t+(n+1)|x-y|} \geq \frac{t}{t+l c|x-y|} .
$$

Eq. (4.1) leads to $\frac{2 n+3}{n+1}\left(1+x^{2}\right)(1+y) \geq \frac{1}{l c}$, that holds for every $l, c \in(0,1)$. Hence $T_{n}$ is $\varphi$-contractive. On the other hand we have:

$$
T=\lim _{n \rightarrow \infty} T_{n}=\lim _{n \rightarrow \infty} \frac{(n+1) x}{(2 n+3)\left(1+x^{2}\right)}=\frac{x}{2\left(1+x^{2}\right)} .
$$

Similarly to the above method, we can see that $T$ is also $\varphi$-contractive. Therefore, Theorem 3.5 holds and the iterative scheme:

$$
x_{n+1}=\frac{(n+1) x_{n}}{(2 n+3)\left(1+x_{n}^{2}\right)}
$$

converges to the unique fixed point of $T$. It is easy to check out that $x^{*}=0$ is the fixed point of $T$. Figure 1 shows the evolution of the iterative scheme (4.2) for different initial conditions. In Figure 1, we can observe that the sequence $\left\{x_{n}\right\}$ converges to zero as predicted by Theorem 3.5. 


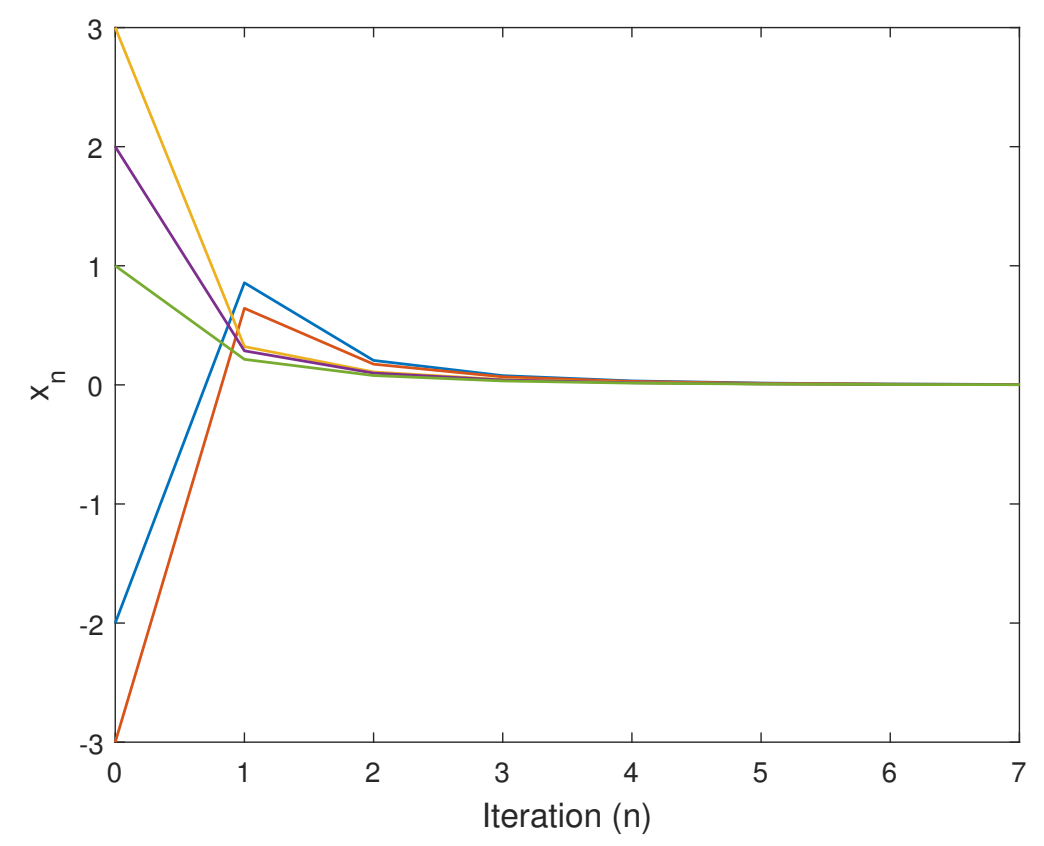

FIGURE 1. Evolution of the sequence of iterates for different initial conditions

\section{Conclusions}

This paper has introduced the concept of $\varphi$-contractive maps in probabilistic modular spaces. Furthermore, the existence of fixed points for these operators in probabilistic modular spaces is investigated as well. Afterwards, the results are extended to a finite linear combination of $\varphi$-contractive mappings. Finally, we also investigate some convergence properties of sequences constructed by these operators which are either convergent to either a $\varphi$-contractive map.

Conflicts of Interest: The author(s) declare that there are no conflicts of interest regarding the publication of this paper.

\section{REFERENCES}

[1] A. B. Amar, A. Jeribi, M. Mnif, Some fixed point theorems and application to biological model, Numer. Funct. Anal. Optim. 29 (1-2) (2008), 1-23.

[2] L. Marks, Fixed-point optimization of atoms and density in DFT, J. Chem. Theory Comput. 9 (6) (2013), 2786-2800.

[3] M. A. Khamsi, W. A. Kirk, An introduction to metric spaces and fixed point theory, vol. 53, Wiley, New York, 2011.

[4] L. B. Ćirić, B. Fisher, Fixed Point Theory: contraction mapping principle, Faculty of Mechanical Engineering, University of Belgrade, Serbia, 2003.

[5] Z. Zuo, Some fixed point property for multivalued nonexpansive mappings in banach spaces, Math Inequal Appl. 7 (1) (2013), 129-137.

[6] K. Menger, Statistical metrics, Proc. Nat. Acad. Sci. 28 (12) (1942), 535-537.

[7] M. Abtahi, Common fixed point theorems of Meir-keeler type in metric spaces, Fixed Point Theory. 1 (2017), 47-56. 
[8] H. Nakano, Modular Semi-Ordered Spaces, Maruzen Co. Ltd., Tokyo, Japan, 1950.

[9] J. Musielak, W. Orlicz, On modular spaces, Stud. Math. 18 (1) (1959) 49-65.

[10] P. Kumam, Fixed point theorems for nonexpansive mappings in modular spaces, Arch. Math. 40 (4) (2004), $345-353$.

[11] K. Kuaket, P. Kumam, Fixed points of asymptotic pointwise contractions in modular spaces, Appl. Math. Lett. 24 (11) (2011), 1795-1798.

[12] M. De la Sen, D. O'Regan, R. Saadati, Characterization of modular spaces, J. Comput. Anal. Appl. 22 (2017), $558-572$.

[13] K. Fallahi, K. Nourouzi, Probabilistic modular spaces and linear operators, Acta Appl. Math. 105 (2) (2009), 123-140.

[14] A. N. Sherstnev, On the notion of a random normed space, Dokl. Akad. Nauk SSSR. 149 (1963), 280-283.

[15] F. Lael, K. Nourouzi, Fixed points of mappings defined on probabilistic modular spaces, Bull. Math. Anal. Appl. 4 (3) (2012), 23-28.

[16] X. Wang, C. Zhang, Y. Cui, Some sufficient conditions for points of multivalued nonexpansive mappings in banach spaces, Math. Inequal. Appl. 11 (1) (2017), 113-120. 